\title{
A Renormalization Group Proof of Perturbative Renormalizability
}

\author{
T. R. Hurd $\star$
}

Department of Mathematics, University of British Columbia, Vancouver, Canada, V6T 1Y4

\begin{abstract}
This paper presents a proof of bounds on the renormalized perturbation expansion of the euclidean $\lambda \phi_{4}^{4}$ theory. Its aim is partly pedagogical: by combining the insights and techniques of numerous authors it is now possible to define the perturbation expansion and bound it in a very few pages. The present version is based on the renormalized tree expansion adapted to the continuous renormalization group : all detailed results are proved by induction on the size of the tree. The continuous RG version presented here has one big advantage over the discrete RG version discussed elsewhere. In the continuous version, a tree has a more restrictive structure: there is a one-to-one correspondence between forks of the tree and lines of Feynman graphs. This extra structure eliminates the need to introduce Feynman graphs in the first place. It also reduces the number of cases to be analyzed at a given inductive step and simplifies the combinatorical estimates.
\end{abstract}

\section{Introduction}

It is recognized by now that renormalization is best understood in the framework of Wilson's renormalization group $[12,13]$. This is well exhibited in the setting of constructive quantum field theory by the resent work Gawedzki and Kupiainen and Feldman et al. on the Gross-Neveu ${ }_{2}$ and infra-red $\phi_{4}^{4}$ models $[8,6]$. Another important and perhaps simpler realization of RG ideas has been in renormalized perturbation theory.

The traditional approach to renormalization theory, highlighted in such landmark papers as $[3,1,11,9,14,2]$, has been based on Feynman graphs and the idea that infinities can be cancelled by the introduction of infinite counterterms into the lagrangian. The most refined formulation of the renormalized Feynman graph expansion was the Zimmermann forest formula, which defines the renormalization

\footnotetext{
* Research supported by the Natural Sciences and Engineering Research Council Present adress: Department of Mathematics and Statistics, McMaster University, Hamilton, Ontario, Canada L8S 4K1
} 
prescription, and when combined with the notion of Hepp sector and the power counting theorems, leads to an ultra-violet convergence proof.

The RG approach to perturbation theory arose in its discrete version independently in $[7,5]$ and in its continuous version in [10]. In the discrete version developed by Gallavotti and Nicolò and refined in [4], the free field propagator is decomposed as a sum over scales:

$$
C(x, y)=\sum_{h=0}^{\Lambda} C^{(h)}(x, y),
$$

where in an appropriate sense, each term $C^{(h)}$ has length scale $M^{-h}, M>1$ being a fixed scale factor and $\Lambda$ is an ultraviolet cutoff. Then, a family of effective potentials $\left\{V_{r}\right\}_{r=-1,0,1, \ldots}$ is a collection of functions of the field $\phi$ which satisfies the following recurrence for each $r \geqq 0$ :

$$
V_{r-1}(\phi)=\log \left[\frac{\int d \phi^{\prime} e^{-\left(\phi^{\prime},\left(C^{(r)}\right)^{-1} \phi^{\prime}\right) / 2} e^{V_{r}\left(\phi^{\prime}+\phi\right)}}{\int d \phi^{\prime} e^{-\left(\phi^{\prime},\left(C^{(r)}\right)^{-1} \phi^{\prime}\right) / 2} e^{V_{r}\left(\phi^{\prime}\right)}}\right] .
$$

This equation can be written as an infinite series in two ways:

$$
\begin{aligned}
V_{r-1}= & V_{r}+\sum_{n=2}^{\infty} \mathscr{E}_{(r)}^{T}\left(V_{r}, \ldots, V_{r}\right), \\
& (n \text { arguments) }, \quad \text { Wick-ordered version ; } \\
V_{r-1}= & V_{r}+\sum_{n=1}^{\infty} \widetilde{\mathscr{E}}_{(r)}^{T}\left(V_{r}, \ldots, V_{r}\right),
\end{aligned}
$$

( $n$ arguments), non-Wick-ordered version ;

where $\mathscr{E}^{T}, \mathscr{E}^{T}$ are called truncated expectations. The renormalized effective potentials are a particular solution of this recurrence which depends uniformly on the cutoff $\Lambda$ as $\Lambda \rightarrow \infty$. Each effective potential is defined by an expansion whose terms are represented pictorially by trees. In the Wick-ordered form, trees have forks with $n \geqq 2$ branches emanating upwards (called $n$-forks), while in the non-Wick ordered form, forks have $n \geqq 1$ upward branches. The value of each tree $\tau$ is calculated as a sum of Feynman graphs each labelled with a nested family of subgraphs compatibly with the tree. At each subgraph is applied either a renormalization operation or a counterterm operation. $\Lambda$-uniform bounds on each tree of the expansion are proved by induction of the number of forks of the tree.

The method described in this paper is a simplification of the above procedure. The original observation which motivated the work was that the tree expansion simplifies as the scale factor $M \rightarrow 1$. Suppose, for example, that $\tau$ is a tree which has a 3-fork $f$, and that $\tau^{\prime}$ is the related tree obtained by splitting $f$ into two 2-forks. For $M=1+\varepsilon$ with $\varepsilon \ll 1$, and any graph $G$ compatible with $\tau$, we expect that

$$
\left\|G_{\tau}\right\| \leqq O(\varepsilon)\left\|G_{\tau^{\prime}}\right\|
$$

Similarly, as $M \rightarrow 1$, the contribution from trees with $n$-forks, $n>2$, ought to become negligible compared to that from trees with only 1,2-forks. This intuition is shown concretely by the fact that (13), the differential analogue of (4), contains only $n=1,2$ terms. Thus the continuous RG tree expansion is over trees with only 1 and 2 forks. 
The simplification is quite dramatic: in the non-Wick-ordered version we adopt here, for any Feynman graph $G$ contributing to a given tree $\tau$, each line $G$ is in oneto-one correspondence with a fork of $\tau$. It turns out that Feynman graphs are superfluous in this framework, and the analysis we present makes no use of them.

The method combines aspects of the GN approach with the approach of Polchinski. It retains the conceptual elegance of the tree expansion, while realizing the goal of freeing the analysis from Feynman graphs, as in Polchinski.

The paper is organized as follows. In Sect. 2, the unrenormalized effective potentials are introduced, and the continuous-scale tree expansion is defined. The natural bound on the value of a completely convergent tree (Theorem 2 ) is proved in Sect. 3. Renormalization operations are introduced in Sect. 4, and the renormalized effective potentials defined in terms of them. The renormalized tree expansion (Theorem 3) is proved. Finally, in Sect. 5, the inductive bound on the value of a renormalized tree is proved (Theorem 6). This is the main result of the paper.

\section{The Unrenormalized Tree Expansion}

Consider Euclidean free field theory in four dimensions with scalar field $\phi(x)$, gaussian measure $d P(\phi)$, and covariance

$$
C(x, y) \equiv \int d P(\phi) \phi(x) \phi(y)=\left(-\partial^{2}+m^{2}\right)^{-1}(x, y) .
$$

This function is singular at $|x-y|=0$. If $m^{2}=0$, it also exhibits slow decay at large separations. We decompose the covariance as an "integral over scales". Several schemes are possible: I choose the following formula:

with

$$
C(x, y)=\int_{0}^{\infty} d \zeta c(x, y, \zeta)
$$

$$
c(x, y, \zeta) \equiv 4 \pi^{2} \exp \left[-\zeta(x-y)^{2}-\frac{m^{2}}{4 \zeta}\right] .
$$

Here we consider only the massive theory: for convenience I take $m^{2}=4$. For any fixed pair of numbers $0 \leqq r<s<\infty$, the cutoff covariance

$$
C_{r}^{s} \equiv \int_{r}^{s} d \zeta c(\zeta)
$$

is $C^{\infty}$ and has exponential large distance decay. Let $d P_{r}^{s}(\phi)$ be the corresponding gaussian measure.

An interacting theory can be defined in the presence of a UV cutoff $\Lambda$ by a bare potential $V(\phi)$, a local functional of $\phi$. The generating functional for connected Green's functions (with external lines amputated by $C_{0}^{\Lambda^{-1}}$ ) is given by

$$
V_{0}^{\Lambda}(\phi)=\log \left[\left\{Z_{0}^{\Lambda}\right\}^{-1} \int d P_{0}^{\Lambda}\left(\phi^{\prime}\right) e^{V\left(\phi^{\prime}+\phi\right)}\right],
$$

where $Z_{0}^{\Lambda}=\int d P_{0}^{\Lambda}\left(\phi^{\prime}\right) e^{V\left(\phi^{\prime}\right)}$. The functional $V_{0}^{\Lambda}$ is called the (unrenormalized) effective potential for the model (n.b. the label "effective potential" is often applied to the generating functional for one-particle irreducible amputated Green's functions). 
The RG approach introduces a one-parameter family of effective potentials $V_{r}^{A}$ which interpolates between $V$ and $V_{0}^{A}$ :

$$
V_{r}^{\Lambda}(\phi)=\log \left[\left\{Z_{r}^{\Lambda}\right\}^{-1} \int d P_{r}^{\Lambda}\left(\phi^{\prime}\right) e^{V\left(\phi^{\prime}+\phi\right)}\right] .
$$

The normalization factor $Z_{r}^{\Lambda}$ is infinite in infinite volume: however, the normalized quantities (11) are easily interpreted by imposing and then removing a volume cutoff. We note $V_{\Lambda}^{\Lambda}=V$ and the "semi-group" property:

$$
V_{r}^{\Lambda}(\phi)=\log \left[\left\{Z_{r}^{s}\right\}^{-1} \int d P_{r}^{s}\left(\phi^{\prime}\right) e^{V_{s}^{\Lambda}\left(\phi^{\prime}+\phi\right)}\right]
$$

which holds for any $s, r \leqq s \leqq \Lambda$.

The dependence of $V_{r}^{\Lambda}$ on the lower cutoff $r$ is characterized by the following differential equation:

Lemma 1. For any $0 \leqq r \leqq \Lambda$ :

$$
\frac{\partial V_{r}^{\Lambda}(\phi)}{\partial r}=-\left[B_{r}^{(1)}\left(V_{r}^{\Lambda}\right)+B_{r}^{(2)}\left(V_{r}^{\Lambda}, V_{r}^{\Lambda}\right)\right]
$$

where $B_{r}^{(1)}$ is the linear operator

$$
B_{r}^{(1)}(W) \equiv \frac{1}{2} \int d x d y c(x, y, r)\left\{\frac{\partial^{2} W}{\partial \phi(x) \delta \phi(y)}-\left.\left[\frac{\partial^{2} W}{\delta \phi(x) \delta \phi(y)}\right]\right|_{\phi=0}\right\},
$$

and $B_{r}^{(2)}$ is the symmetric bilinear operator

$$
B_{r}^{(2)}(V, W) \equiv \frac{1}{2} \int d x d y c(x, y, r)\left\{\frac{\partial V}{\delta \phi(x)} \frac{\delta W}{\delta \phi(y)}-\left.\left[\frac{\delta V}{\delta \phi(x)} \frac{\delta W}{\delta \phi(y)}\right]\right|_{\phi=0}\right\}
$$

acting on functionals of $\phi$.

Proof. We approximate $F_{r} \equiv \exp V_{r}$ by finite dimensional integrals of the form

$$
\begin{aligned}
F_{r, n}(\phi) & =\left[Z_{r, n}\right]^{-1} \int d \phi^{\prime} e^{-\left(\phi^{\prime}, C_{r, n}^{-1} \phi^{\prime}\right) / 2} e^{V\left(\phi^{\prime}+\phi\right)}, \\
Z_{r, n} & =\int d \phi^{\prime} e^{-\left(\phi^{\prime}, C_{r, n}^{-1} \phi^{\prime}\right) / 2} e^{V\left(\phi^{\prime}\right)},
\end{aligned}
$$

and calculate

$$
\begin{aligned}
\frac{\partial F_{r, n}}{\partial r}= & 1 / 2\left[Z_{r, n}\right]^{-1} \int d \phi^{\prime}\left(C_{r, n}^{-1} \phi^{\prime}, c_{r, n} C_{r, n}^{-1} \phi^{\prime}\right) e^{-\left(\phi^{\prime}, C_{r, n}^{-1} \phi^{\prime}\right) / 2} e^{V\left(\phi^{\prime}+\phi\right)}, \\
& +1 / 2 F_{r, n}\left[Z_{r, n}\right]^{-1} \int d \phi^{\prime}\left(C_{r, n}^{-1} \phi^{\prime} c_{r, n} C_{r, n}^{-1} \phi^{\prime}\right) e^{-\left(\phi^{\prime}, C_{r, n}^{-1} \phi^{\prime}\right) / 2} e^{V\left(\phi^{\prime}\right)} .
\end{aligned}
$$

After twice integrating by parts, we find

$$
\frac{\partial F}{\partial r}=-1 / 2 \int d x d y c(x, y, r)\left\{\frac{\delta^{2} F}{\delta \phi(x) \delta \phi(y)}-\left.F\left[\frac{\delta^{2} F}{\delta \phi(x) \delta \phi(y)}\right]\right|_{\phi=0}\right\}
$$

Now, use the chain rule on $F=e^{V}$, and find

$$
\frac{\partial e^{V}}{\partial r}=e^{V} \frac{\partial V}{\partial r}=-e^{V}\left[B^{(1)}(V)+B^{(2)}(V, V)\right] \text {. QED }
$$


By the fundamental theorem of calculus,

$$
V_{r}^{\Lambda}(\phi)=V(\phi)+\int_{r}^{\Lambda} d \zeta\left[B_{\zeta}^{(1)}\left(V_{\zeta}^{\Lambda}\right)+B_{\zeta}^{(2)}\left(V_{\zeta}^{\Lambda}, V_{\zeta}^{\Lambda}\right)\right] .
$$

The unrenormalized tree expansion is the formal power series solution of this integral equation obtained by iteration. We represent each term of the expansion

$$
\begin{aligned}
V_{r}^{\Lambda}(\phi)= & V(\phi)+\int_{r}^{A} d \zeta\left[B_{\zeta}^{(1)}\left(V+\int_{\zeta}^{A} d \zeta^{\prime}\left[B_{\zeta^{\prime}}^{(1)}(V+\cdots)+B_{\zeta^{\prime}}^{(2)}(V+\cdots, V+\cdots)\right]\right)\right. \\
& \left.+B_{\zeta}^{(2)}(V+\cdots, V+\cdots)\right]
\end{aligned}
$$

by a tree:

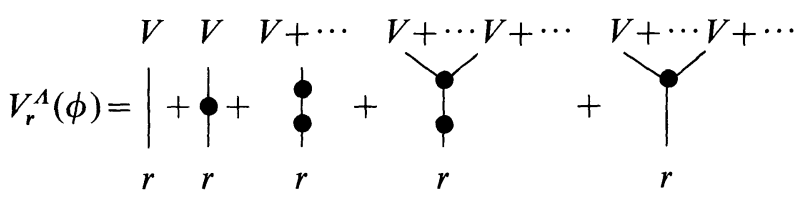

Each term is a planted planar tree which has (i) a number $v(\tau)$ of upper endpoints, which represent the bare potential $V$; (ii) a number $N(\tau)=N_{1}(\tau)+N_{2}(\tau)$ of nodes ("forks") with either one or two "branches" emanating upwards, and one branch emanating downwards; and (iii) the label $r$ at the "root". Each fork represents either the operator $\int d \zeta B_{\zeta}^{(1)}(\cdot)$ or $\int d \zeta B_{\zeta}^{(2)}(\cdot, \cdot)$. All such trees occur in the sum (22) exactly once. The unrenormalized tree expansion can be written:

$$
V_{r}^{\Lambda}(\phi)=\sum_{\tau} v_{r}^{\Lambda}(\tau, \phi)=\sum_{\tau} \int_{\mathscr{Z}_{r}^{\Lambda}(\tau)}\left[\prod_{f \in \mathscr{F}(\tau)} d \zeta_{f}\right] v(\tau, \zeta, \phi) .
$$

Here $\mathscr{F}(\tau)$ denotes the set of forks of the tree $\tau$, and $\mathscr{Z}_{r}{ }^{\Lambda}(\tau)$ denotes the domain:

$$
\left\{\begin{array}{l}
\zeta_{\pi(f)} \leqq \zeta_{f} \leqq \Lambda, \\
r \leqq \zeta_{F} \leqq \Lambda
\end{array}\right.
$$

where $F$ is the bottom fork of $\tau$ and $\pi(f)$ denotes the fork immediately beneath the fork $f$. We will also write $f<f^{\prime}$ if $f^{\prime}$ lies above $f, \tau_{\geqq f}$ for the tree with forks $\mathscr{F}(\tau) \cap\left\{f^{\prime}: f^{\prime} \geqq f\right\}$, etc.

The value $v_{r}^{\Lambda}(\tau, \phi)$ of a given tree is defined by induction down the forks of the tree. Thus, $v_{r}^{\Lambda}(\tau, \phi)=V(\phi)$ if $\tau$ is trivial,

$$
v_{r}^{\Lambda}(\tau, \phi)=\int_{r}^{\Lambda} d \zeta_{F} B_{\zeta_{F}}^{(2)}\left(\left(v_{\zeta_{F}}^{\Lambda}\left(\tau_{1}, \phi\right), v_{\zeta_{F}}^{\Lambda}\left(\tau_{2}, \phi\right)\right),\right.
$$

if $F$ is a 2 -fork with trees $\tau_{1}$ and $\tau_{2}$ emanating upwards, and

$$
v_{r}^{\Lambda}(\tau, \phi)=\int_{r}^{A} d \zeta_{F} B_{\zeta_{F}}^{(1)}\left(v_{\zeta_{F}}^{\Lambda}\left(\tau_{1}, \phi\right)\right)
$$

if $F$ is a 1 -fork with tree $\tau_{1}$ emanating upwards. 
It is useful to work with kernels. Let $v(\phi)$ be a monomial of degree $d$. It can be written

where

$$
v(\phi)=\int\left[\prod_{i=1}^{d} d x_{i} \phi\left(x_{i}\right)\right] v\left(x_{1}, \ldots, x_{d}\right),
$$

$$
\left.v\left(x_{1}, \ldots, x_{d}\right) \equiv \frac{1}{d !}\left[\prod_{i=1}^{d} \frac{\delta}{\delta \phi\left(x_{i}\right)}\right] v(\phi)\right|_{\phi=0} .
$$

We introduce a compact notation $\mathbf{x}=\left(x_{1}, \ldots, x_{d}\right), \quad d \mathbf{x}=d x_{1} \cdots d x_{d}, \quad \phi(\mathbf{x})$ $=\phi\left(x_{1}\right) \cdots \phi\left(x_{d}\right), \delta / \delta \phi(\mathbf{x})=\delta / \delta \phi\left(x_{1}\right) \cdots \delta / \delta \phi\left(x_{d}\right)$, etc. The operations $B_{r}^{(i)}$ can be expressed in terms of kernels. Suppose $v_{1}, v_{2}$ have degrees $d_{1}$ and $d_{2}$. Then $v=B_{r}^{(2)}\left(v_{1}, v_{2}\right)$ has degree $d=d_{1}+d_{2}-2$ and kernel

$$
\begin{aligned}
v(\mathbf{x}) & =\left.\frac{1}{2} \int d y d z c(y, z, r)\left\{\frac{1}{d !} \frac{\delta^{d}}{\delta \phi(\mathbf{x})}\left[\frac{\delta v_{1}(\phi)}{\delta \phi(y)} \frac{\delta v_{2}(\phi)}{\delta \phi(z)}\right]\right\}\right|_{\phi=0} \\
& =\frac{d_{1} ! d_{2} !}{d !} \sum_{\Pi=\left\{\pi_{1}, \pi_{2}\right\}} \frac{1}{2} \int d y d z c(y, z, r) v_{1}\left(\mathbf{x}_{\pi_{1}}, y\right) v_{2}\left(\mathbf{x}_{\pi_{2}}, z\right),
\end{aligned}
$$

where $\Pi$ is a partition of $\{1, \ldots, d\}$ into subsets of size $d_{1}-1$ and $d_{2}-1$. The monomial $v^{\prime}=B_{r}^{(1)}\left(v_{1}\right)$ has degree $d=d_{1}-2$ and kernel

$$
v^{\prime}(\mathbf{x})=\frac{d_{1} !}{d !} \frac{1}{2} \int d y d z c(y, z, r) v_{1}(\mathbf{x}, y, z)
$$

\section{Bounds on the Unrenormalized $\lambda \phi^{\mathbf{4}}$ Expansion}

We now consider the value of a typical tree $\tau$ when the bare potential is the local monomial

$$
V(\phi) \equiv-\lambda \int d x(\phi(x))^{4}
$$

We are in particular interested in the dependence of $v_{r}^{\Lambda}(\tau, \phi)$ as the cutoff $\Lambda$ goes to infinity.

The quantity $v_{r}^{\Lambda}(\tau, \phi)$ is easily seen to be a monomial of degree

$$
d_{\tau}=4 v(\tau)-2\left(N_{1}(\tau)+N_{2}(\tau)\right)
$$

A suitable measure of the size of $v_{r}^{\Lambda}(\tau, \phi)$ is the following norm on the kernel:

$$
\left\|v_{r}^{\Lambda}(\tau)\right\| \equiv \int \prod_{i=1}^{d_{\tau}} d x_{i} \delta\left(x_{1}\right)\left|v_{r}^{\Lambda}(\tau, \mathbf{x})\right| .
$$

(The kernel $v_{r}^{A}$ in general involves undifferentiated delta functions, for which we define $|\delta|=\delta$.) If $\left\|v_{r}^{\Lambda}(\tau)\right\|$ is bounded uniformly in $\Lambda$ we say that the tree is convergent, otherwise it is called divergent. If $v_{r}^{\Lambda}(\tau)$ is convergent, then $\lim v_{r}^{\Lambda}(\tau, \mathbf{x})$ exists and is a locally integrable distribution which is bounded $\Lambda \rightarrow \infty$ at $\infty$. 
We consider the tree $\tau$ in the two cases $F \in \mathscr{F}^{(2)}, F \in \mathscr{F}^{(1)}$. If $F \in \mathscr{F}^{(2)}$, then from (25) and (29) we obtain the natural bound on $\left\|v_{r}^{\Lambda}(\tau)\right\|$ :

$$
\left\|v_{r}^{\Lambda}(\tau)\right\| \leqq \frac{d_{\tau_{1}} d_{\tau_{2}}}{2} \int_{r}^{\Lambda} d \zeta\|c(\zeta)\|\left\|v_{\zeta}^{\Lambda}\left(\tau_{1}\right)\right\|\left\|v_{\zeta}^{\Lambda}\left(\tau_{2}\right)\right\| .
$$

The norm $\|c(\zeta)\|$ can be evaluated explicitly:

and so

$$
\|c(\zeta)\|=e^{-1 / \zeta} \int d^{4} y e^{-\zeta y^{2}}=\pi^{2} e^{-1 / \zeta \zeta^{-2}},
$$

$$
\left\|v_{r}^{\Lambda}(\tau)\right\| \leqq \frac{d_{\tau_{1}} d_{\tau_{2}} \pi^{2}}{2} \int_{r}^{\Lambda} d \zeta e^{-1 / \zeta} \zeta^{-2}\left\|v_{\zeta}^{\Lambda}\left(\tau_{1}\right)\right\|\left\|v_{\zeta}^{\Lambda}\left(\tau_{2}\right)\right\| .
$$

If $F \in \mathscr{F}^{(1)}$, then from (26) and (30) we obtain the bound on $\left\|v_{r}^{\Lambda}(\tau)\right\|$ :

$$
\begin{aligned}
\left\|v_{r}^{\Lambda}(\tau)\right\| & \leqq \frac{d_{\tau_{1}}\left(d_{\tau_{1}}-1\right)}{2} \int_{r}^{\Lambda} d \zeta\left[\sup _{y, z}|c(y, z, \zeta)|\right]\left\|v_{\zeta}^{\Lambda}\left(\tau_{1}\right)\right\| \\
& \leqq \frac{d_{\tau_{1}}\left(d_{\tau_{1}}-1\right)}{2} \int_{r}^{\Lambda} d \zeta e^{-1 / \zeta}\left\|v_{\zeta}^{\Lambda}\left(\tau_{1}\right)\right\| .
\end{aligned}
$$

We introduce the superficial degree of divergence at the fork $f$ :

$$
\delta_{f} \equiv 2\left(N_{1}(f)-N_{2}(f)\right),
$$

where $N_{i}(f) \equiv N_{i}\left(\tau_{\geqq f}\right)$, and, when $\delta_{f}<0$ at each fork $f$, a combinatoric factor defined inductively:

$$
c(\tau)= \begin{cases}1 & \tau \text { trivial } \\ d_{\tau_{1}}\left(d_{\tau_{1}}-1\right)\left|\delta_{F}\right|^{-1} c\left(\tau_{1}\right) & F \in \mathscr{F}^{(1)} \\ \pi^{2} d_{\tau_{1}} d_{\tau_{2}}\left|\delta_{F}\right|^{-1} c\left(\tau_{1}\right) c\left(\tau_{2}\right) & F \in \mathscr{F}^{(2)}\end{cases}
$$

Theorem 2. Suppose $\tau$ is such that $\delta_{f}<0$ for all forks $f \in \mathscr{F}$. Then $v_{r}^{\Lambda}(\tau, \phi)$ is convergent. It satisfies the A-uniform bounds:

$$
\left\|v_{r}^{\Lambda}(\tau)\right\| \leqq \lambda^{v} c(\tau) E(r)^{-\delta_{F} / 2}
$$

where $E(r) \equiv\left(1-e^{-1 / r}\right)$.

Remarks. (i) When $\delta_{f} \geqq 0$ at any fork, it can be shown in this model that $v_{r}^{\Lambda}$ diverges as $\Lambda \rightarrow \infty$.

(ii) The proof and the renormalized generalization which follows make use of a certain elegant property of the integrals arising here: to evaluate:

$$
I(r)=\int_{r}^{\infty} d \zeta e^{-1 / \zeta} \zeta^{-2} E(\zeta)^{\alpha-1}, \quad(\alpha>0)
$$

we make the change of variables (good for all $\zeta \in(0, \infty)) \zeta \mapsto x(\zeta)=\log \left(E(\zeta)^{-1}\right)$.

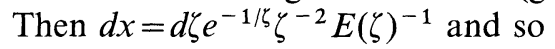

$$
I(r)=\int_{x(r)}^{\infty} d x e^{-\alpha x}=-\left.\frac{1}{\alpha} e^{-\alpha x}\right|_{x(r)} ^{\infty}=\frac{1}{\alpha} E(r)^{\alpha} .
$$


(iii) We do not discuss here the behaviour of the combinatoric factor $c(\tau)$ nor the behaviour of the sum over trees: this is the subject for further investigation. Let it suffice to give here the following worst-case bound for a tree $\tau$ with $N_{i} i$-forks, $i=1,2$ :

$$
|c(\tau)| \leqq 4 \cdot 10^{N_{1}}\left(3 \sqrt{32} \pi^{2}\right)^{N_{2}}\left(N_{2}+2\right) !^{1 / 2} N_{1} ! .
$$

Proof. We prove (40) for the tree $\tau$ by the inductive use of (36) and (37). Clearly (40) holds for the trivial tree. Suppose $F \in \mathscr{F}^{(2)}$. Assuming (40) for the trees $\tau_{1}, \tau_{2}$, then (36) implies

$$
\left\|v_{r}^{\Lambda}(\tau)\right\| \leqq \frac{d_{\tau_{1}} d_{\tau_{2}} c\left(\tau_{1}\right) c\left(\tau_{2}\right) \pi^{2}}{2} \int_{r}^{\Lambda} d \zeta e^{-1 / \zeta} \zeta^{-2} E(\zeta)^{-\left(\delta_{1}+\delta_{2}\right) / 2} .
$$

Note that $\delta_{1}+\delta_{2}=\delta_{F}+2$ : Provided $\delta_{F}<0$, we can do the $\zeta$-integral using (42) and obtain the desired bound. Suppose $F \in \mathscr{F}^{(1)}$. Then

$$
\left\|v_{r}^{\Lambda}(\tau)\right\| \leqq \frac{d_{\tau_{1}}\left(d_{\tau_{1}}-1\right) c\left(\tau_{1}\right)}{2} \int_{r}^{\Lambda} d \zeta e^{-1 / \zeta} E(\zeta)^{-\delta_{F} / 2+1} .
$$

Since $\zeta E(\zeta) \leqq 1$ for all $0 \leqq \zeta<\infty$, we can write $E(\zeta)^{-\delta_{F} / 2+1} \leqq \zeta^{-2} E(\zeta)^{-\delta_{F} / 2-1}$ and then (42) gives the desired bound, again provided $\delta_{F}<0$. QED

\section{The Renormalized Tree Expansion}

We introduce a localization operator $L$ acting on the vector space of effective potentials, which projects onto the subspace of relevant monomials. Heuristically (the exact definition will be given shortly), the relevant monomials are those monomials for which the convergence condition fails (so $\delta \geqq 0$ ), while irrelevant monomials are those with $\delta<0$. We use $L$ and its orthogonal projection $R=1-L$ to formally define the following solution of the ode (13):

$$
\begin{aligned}
V_{r}^{\Lambda, \text { ren }}= & V+\int_{r}^{A} d \zeta R\left[B_{\zeta}^{(1)}\left(V_{\zeta}^{\Lambda, \text { ren }}\right)+B_{\zeta}^{(2)}\left(V_{\zeta}^{\Lambda, \text { ren }}, V_{\zeta}^{\Lambda, \text { ren }}\right)\right] \\
& -\int_{0}^{r} d \zeta L\left[B_{\zeta}^{(1)}\left(V_{\zeta}^{\Lambda, \text { ren }}\right)+B_{\zeta}^{(2)}\left(V_{\zeta}^{\Lambda, \text { ren }}, V_{\zeta}^{\Lambda, \text { ren }}\right)\right] .
\end{aligned}
$$

We check that

$$
\left\{\begin{array}{l}
L V_{0}^{\Lambda, \text { ren }}=L V=V, \\
R V_{\Lambda}^{\Lambda, \text { ren }}=R V=0,
\end{array}\right.
$$

since $V=-\lambda \int \phi^{4}$ is itself "relevant". We call (46) the renormalized effective potential at scale $r$ : it is the solution of (13) which satisfies a mixed boundary condition: the relevant part of $V_{r}$ is fixed at scale 0 , while the irrelevant part of $V_{r}$ is set equal to zero at scale $\Lambda$.

The renormalized tree expansion for $V_{r}^{\Lambda, \text { ren }}$ is the formal power series solution obtained from (46) by iteration. It is an expansion like (23), with two changes: (i) each fork is assigned a label $\varrho_{f}$ which is either $R$ or $C$, signifying the insertion of an 
$R$ or $C \equiv-L$ operator there, and (ii) the domain of the $\zeta$-integrals is the space $\mathscr{Z}_{r}^{\Lambda}(\tau, \varrho)$ defined by

$$
\begin{cases}\zeta_{\pi(f)} \leqq \zeta_{f} \leqq \Lambda, & \text { if } \varrho_{f}=R \\ 0 \leqq \zeta_{f} \leqq \zeta_{\pi(f)}, & \text { if } \varrho_{f}=C ;\end{cases}
$$

with $\zeta_{\pi(F)} \equiv r$ for the bottom fork $F$. The value $v(\tau, \varrho, \zeta, \phi)$ is defined inductively as in (25) and (26), but with the operation $\varrho_{f}$ (either $R$ or $C$ ) applied at each fork $f$. The expansion is then

Theorem 3. (Renormalized tree expansion).

$$
V_{r}^{\Lambda, \text { ren }}(\phi)=\sum_{\tau} \sum_{\varrho} v_{r}^{\Lambda, \text { ren }}(\tau, \varrho, \phi)=\sum_{\tau} \sum_{\varrho} \int_{\mathscr{Z}_{r}^{\Lambda}(\tau, \varrho)} d \zeta v(\tau, \varrho, \zeta, \phi) .
$$

The value $v(\phi)$ of a tree which has renormalization at every fork except the bottom fork will turn out to depend explicitly not only on the field $\phi$, but also on the first, second, and third derivatives which we write $\phi_{\mu} \equiv \partial_{\mu} \phi$, $\phi_{\mu \nu} \equiv \partial_{\mu} \partial_{\nu} \phi, \phi_{\mu v \varrho} \equiv \partial_{\mu} \partial_{\nu} \partial_{\varrho} \phi$. The total degree of $v$ in $\phi$ is $d=4-2\left(N_{1}-N_{2}\right)$, as before, but now $v$ has many terms:

$$
v(\phi)=\sum_{\mathbf{n}=\left\{n_{1}, \ldots, n_{d}\right\}} \int d x_{1} \cdots d x_{d} \phi_{n_{1}}\left(x_{1}\right) \cdots \phi_{n_{d}}\left(x_{d}\right) v_{n_{1} \ldots n_{d}}\left(x_{1}, x_{d}\right),
$$

where each $n_{i}$ is a 4-dimensional multiindex of degree $0 \leqq\left|n_{i}\right| \leqq 3$ (i.e. $n_{i}=\emptyset, \mu, \mu v$, or $\mu v \varrho$ where $\mu, v, \varrho=1,2,3$ or 4 ). We write

$$
v(\phi)=\sum_{\mathbf{n}} v_{\mathbf{n}}(\phi)
$$

We introduce a compactified index notation: $X_{i} \equiv\left(x_{i}, n_{i}\right), \phi\left(X_{i}\right) \equiv \phi_{n_{i}}\left(x_{i}\right)$, $\int d X_{i} \equiv \sum_{n_{i}} \int d x_{i}, \quad \mathbf{X}=\left\{X_{1}, \ldots, X_{d}\right\}, \int d \mathbf{X}=\int d X_{1} \cdots d X_{d}, \delta / \delta \phi\left(X_{i}\right) \equiv \delta / \delta \phi_{n_{i}}\left(x_{i}\right)$, etc. Then

and

$$
v(\phi)=\int d \mathbf{X} \phi(\mathbf{X}) v(\mathbf{X})
$$

$$
v(\mathbf{X})=\left.\frac{1}{d !}\left[\frac{\delta^{d} v(\phi)}{\delta \phi(\mathbf{X})}\right]\right|_{\phi=0} .
$$

The operators $B^{(1)}, B^{(2)}$ work as before. Suppose $v_{1}(\phi)$ and $v_{2}(\phi)$ have degrees $d_{1}, d_{2}$. Then $v=B_{r}^{(2)}\left(v_{1}, v_{2}\right)$ has degree $d=d_{1}+d_{2}-2$ and kernels

$$
v(\mathbf{X})=\frac{d_{1} ! d_{2} !}{d !} \sum_{\Pi=\left\{\pi_{1}, \pi_{2}\right\}} \frac{1}{2} \int d Y d Z c(Y, Z, r) v_{1}\left(\mathbf{X}_{\pi_{1}}, Y\right) v_{2}\left(\mathbf{X}_{\pi_{2}}, Z\right) .
$$

Here $Y=(y, p)$ and $Z=(z, q)$, so $c(Y, Z, r)=\partial_{y}^{p} \partial_{z}^{q} c(y, z, r)$. The monomial $v^{\prime}=B_{r}^{(1)}\left(v_{1}\right)$ has degree $d=d_{1}-2$ and kernels

$$
v^{\prime}(\mathbf{X})=\frac{d_{1} !}{d !} \frac{1}{2} \int d Y d Z c(Y, Z, r) v_{1}(\mathbf{X}, Y, Z)
$$

The degree of divergence of $v_{\mathbf{n}}$ now depends on $\mathbf{n}$ rather than just $d$ :

$$
\delta_{\mathbf{n}} \equiv 4-\sum_{i}\left|n_{i}\right|-d
$$


A monomial $v_{\mathbf{n}}$ is said to be relevant if $\delta_{\mathbf{n}} \geqq 0$ and irrelevant otherwise. The list of all relevant values of $\mathbf{n}$ is: $(\emptyset, \emptyset),(\emptyset, \mu),(\mu, v),(\emptyset, \mu v),(\emptyset, \emptyset, \emptyset, \emptyset)$.

The localization operator $L$ acting on $v_{\mathbf{n}}(\phi)$ with $\delta \equiv \delta_{\mathbf{n}} \geqq 0$ is a sum of terms of degree $m$ with $0 \leqq m \leqq \delta$ :

with

$$
\left(L v_{\mathbf{n}}\right)(\phi)=\sum_{m=0}^{\delta}\left(L^{m} v_{\mathbf{n}}\right)(\phi)
$$

$$
\left(L^{m} v_{\mathbf{n}}\right)(\phi)=\left.\frac{1}{(\delta-m) !} \frac{d^{\delta-m}}{d t^{\delta-m}} \int d \mathbf{x} v_{\mathbf{n}}(\mathbf{x}) \phi_{\mathbf{n}}(\mathbf{x}(t))\right|_{t=0},
$$

where $x_{i}(t)=x_{1}+t\left(x_{i}-x_{1}\right)$ for $i=2, \ldots, d . L^{m} v_{\mathrm{n}}$ vanishes if $\delta_{\mathrm{n}}<0$. There is some ambiguity in this definition of $L^{m} v_{\mathbf{n}}$ which is completely removed if we select a localization vertex $x_{1}$ such that $\left|n_{1}\right| \leqq\left|n_{i}\right|$ for all $i$.

The renormalization operator $R$ acting on $v_{\mathbf{n}}$ can be expressed using the Taylor remainder formula :

$$
\left(R v_{\mathbf{n}}\right)(\phi)=\frac{1}{\delta !} \int_{0}^{1} d t(1-t)^{\delta} \frac{d^{\delta+1}}{d t^{\delta+1}} \int d \mathbf{x} v_{\mathbf{n}}(\mathbf{x}) \phi_{\mathbf{n}}(\mathbf{x}(t))
$$

Of course, $R=1$ if $\delta<0$. We note that $R v_{\mathbf{n}}$ is always irrelevant: it is a polynomial $(R v)_{\mathbf{n}^{\prime}}$ whose degree of divergence is $\delta_{\mathbf{n}, \text { ren }} \equiv \delta_{\mathbf{n}^{\prime}}=\min \left(\delta_{\mathbf{n}},-1\right)<0$.

\section{Bounds on the Renormalized Expansion}

We define the norm of $v_{\mathbf{n}}$ as before:

$$
\left\|v_{\mathbf{n}}\right\| \equiv\left\|v_{\mathbf{n}}\right\|_{0}=\int d \mathbf{x} \delta\left(x_{1}\right)\left|v_{\mathbf{n}}(\mathbf{x})\right| .
$$

More generally, for any $\gamma \geqq 0$, we define

$$
\left\|v_{\mathbf{n}}\right\|_{\gamma}=\frac{1}{\gamma !} \sup _{\widetilde{\Delta}^{\gamma}} \int d \mathbf{x} \delta\left(x_{1}\right)\left|\tilde{\Delta}^{\gamma}\right|\left|v_{\mathbf{n}}(\mathbf{x})\right|
$$

where $\tilde{\Delta}^{\gamma}(\mathbf{x})=\prod_{r=1}^{\gamma}\left(x_{i_{r}}-x_{j_{r}}\right)$ is any difference of degree $\gamma \geqq 0$, and the sup in (61) takes place over all choices of indices $i_{r}, j_{r}$. The following lemma gives what we need to know about the size of renormalized kernels.

Lemma 4. If $v_{\mathbf{n}}$ is any monomial with $\delta_{\mathbf{n}} \geqq 0$, then

$$
\begin{cases}\left\|R v_{\mathbf{n}}\right\|_{\gamma} \leqq 3\left\|v_{\mathbf{n}}\right\|_{\gamma+\delta_{\mathbf{n}}+1}, & \text { for any } \gamma \geqq 0 ; \\ \left\|L^{m} v_{\mathbf{n}}\right\|_{0} \leqq\left\|v_{\mathbf{n}}\right\|_{\delta_{\mathbf{n}}-m}, & \text { for } 0 \leqq m \leqq \delta_{\mathbf{n}} \\ \left\|L^{m} v_{\mathbf{n}}\right\|_{\gamma}=0, & \text { for any } \gamma \geqq 1 .\end{cases}
$$

Proof. Consider the $R$ case. Note that the $t$-derivatives generate a maximum of three terms of the form

$$
\frac{1}{\delta !} \int_{0}^{1} d t(1-t)^{\delta} \int d \mathbf{x} v_{\mathbf{n}}(\mathbf{x}) \Delta^{\delta+1} \phi_{\mathbf{n}^{\prime}}(\mathbf{x}(t))
$$


To calculate a bound on $\left\|R v_{\mathbf{n}}\right\|_{\gamma}$ we note that $\tilde{\Delta}^{\gamma}(\mathbf{x}(t))=t^{\gamma} \tilde{\Delta}^{\gamma}(\mathbf{x})$, set each field $\phi_{n_{i}^{\prime}}(x(t))=1$, and find

$$
\left\|R v_{\mathbf{n}}\right\|_{\gamma} \leqq 3 \sup _{\tilde{\Delta}^{\gamma}, \tilde{\Delta}^{\delta+1}}\left[\frac{1}{\gamma ! \delta !} \int_{0}^{1} d t t^{\gamma}(1-t)^{\delta}\right] \int d \mathbf{x}\left|v_{\mathbf{n}}(\mathbf{x})\right|\left|\tilde{\Delta}^{\gamma+\delta+1}(\mathbf{x})\right| .
$$

But $[\gamma ! \delta !]^{-1} \int_{0}^{1} d t t^{\gamma}(1-t)^{\delta}=[(\gamma+\delta+1) !]^{-1}$ and the result follows. The bounds on $L^{m} v_{\mathbf{n}}$ are trivial. QED

Now we consider how to bound the value $v(r, \phi)$ of a tree $\tau$ with root scale $r$, in the cases $F \in \mathscr{F}^{(2)}$ or $\mathscr{F}^{(1)}, \varrho_{F}=R$ or $C^{m}, m=0,2$. If $F \in \mathscr{F}^{(i)}, i=1,2$,

$$
v(r, \phi)= \begin{cases}\frac{1}{2} \int_{r}^{1} d \zeta R B^{(i)}(\zeta, \phi), & \text { if } \varrho_{F}=R ; \\ \frac{1}{2} \int_{0}^{r} d \zeta C^{m} B^{(i)}(\zeta, \phi), & \text { if } \varrho_{F}=C^{m}\end{cases}
$$

where the quantities $B^{(i)}$ are expressed in terms of the values $v_{1}, v_{2}$ of the trees leading into $F$ by Eqs. (54) and (55). If we write $Y=(y, p), Z=(z, q)$ and note that $\#\{\Pi\}=C\left(d, d_{1}-1\right)$, we find

$$
\begin{aligned}
\left\|B_{\mathbf{n}}^{(2)}(\zeta)\right\|_{\gamma} \leqq & d_{1} d_{2} \sup _{\Pi, \tilde{\Delta}^{\gamma}} \sum_{p, q} \int d \mathbf{x} d y d z \\
& \left|\tilde{\Delta}^{\gamma}\left\|\partial^{p+q} c(y, z, \zeta)\right\| v_{1,\left(\mathbf{n}_{\pi_{1}}, p\right)}\left(\zeta, \mathbf{x}_{\pi_{1}}, y\right) \| v_{2,\left(\mathbf{n}_{\pi_{2}}, q\right)}\left(\zeta, \mathbf{x}_{\pi_{2}}, z\right)\right| .
\end{aligned}
$$

A difference $\left|x_{i}-x_{j}\right|$ with $i \in \pi_{1}$ and $j \in \pi_{2}$ can be bounded by

$$
\left|x_{i}-y\right|+|y-z|+\left|z-x_{j}\right| \text {. }
$$

Expanding $\tilde{\Delta}^{\gamma}$ in this way, we have a bound

$$
\left|\tilde{\Delta}^{\gamma}\right| \leqq \sum_{\substack{\text { partitions } \\ \gamma=\left\{\alpha_{1}, \alpha_{2}, \beta\right\}}}\left|\tilde{\Delta}^{\alpha_{1}}\left\|\tilde{\Delta}^{\alpha_{2}}\right\| \tilde{\Delta}^{\beta}\right|,
$$

where $\tilde{\Delta}^{\alpha_{1}}$ contains differences in the variables $\left\{\mathbf{x}_{\pi_{1}}, y\right\}, \tilde{\Delta}^{\alpha_{2}}$ contains differences in the variables $\left\{\mathbf{x}_{\pi_{2}}, z\right\}$, and $\tilde{\Delta}^{\beta}=|y-z|^{\beta}$. From (66) and the multinomial theorem we have

$$
\begin{aligned}
\left\|B_{\mathbf{n}}^{(2)}(\zeta)\right\|_{\gamma} \leqq & d_{1} d_{2} \sup _{\Pi} \sum_{p, q} \sum_{\beta=0}^{\gamma} \sum_{\alpha=0}^{\gamma-\beta} \\
& \left\|\partial^{p+q} c(\zeta)\right\|_{\beta}\left\|v_{1,\left(\mathbf{n}_{\pi_{1}}, p\right)}(\zeta)\right\|_{\alpha}\left\|v_{2,\left(\mathbf{n}_{\pi_{2}}, q\right)}(\zeta)\right\|_{\gamma-\alpha-\beta} .
\end{aligned}
$$

Somewhat more directly, we can calculate from (55) that

$$
\left\|B_{\mathbf{n}}^{(1)}(\zeta)\right\|_{\gamma} \leqq d_{1}\left(d_{1}-1\right) \sum_{p, q}\left[\sup _{y}\left|\partial_{y}^{p+q} c(y, z, \zeta)\right|\left\|v_{1,\left(\mathbf{n}_{\pi_{1}}, p, q\right)}(\zeta)\right\|_{\gamma}\right] .
$$

Our bound on the value of a renormalized tree, as in the unrenormalized case, is proved by induction down the forks of the tree. The difficulty, of course, is in framing the induction with the right inductive form for the bound. From past 
experience, we know that the right inductive bound must extend the unrenormalized version (40) to include the $\gamma$-dependence of $\left\|v_{\mathbf{n}}\right\|_{\gamma}$, and include "renormalon" factors which will arise from the presence of $C$-forks. This renormalon behaviour will be captured by the following family of functions:

$$
\lambda_{n}(r)=\frac{1}{4} E(r)^{-1 / 4} \int_{r}^{\infty} d \zeta e^{-1 / \zeta} \zeta^{-2} E(\zeta)^{-1+1 / 4}\left(\log E(\zeta)^{-1}\right)^{n}
$$

for $n=0,1,2, \ldots$ (which are essentially the same functions is those introduced in [5]). These satisfy the following properties which are useful for the induction:

\section{Lemma 5.}

$$
\lambda_{n} \text { is monotonically increasing ; }
$$

$$
\lambda_{n_{1}}(r) \lambda_{n_{2}}(r) \leqq \lambda_{n_{1}+n_{2}}(r) ;
$$

$$
\begin{gathered}
\int_{r}^{\infty} d \zeta e^{-1 / \zeta} \zeta^{-2} E(\zeta)^{-1+\beta} \lambda_{n}(\zeta)<2 \lambda_{n}(r) \frac{E(r)^{\beta}}{\beta} \text { for all } \beta \geqq 1 / 2 ; \\
\int_{0}^{r} d \zeta e^{-1 / \zeta} \zeta^{-2} E(\zeta)^{-1} \lambda_{n}(\zeta)<(n+1)^{-1} \lambda_{n+1}(r) ; \\
\lambda_{n}(0)=4^{n} n ! .
\end{gathered}
$$

Proof. We work in the variables $x(r)=\log \left(E(r)^{-1}\right)$ in terms of which:

$$
\tilde{\lambda}_{n}(x)=\lambda_{n}(r(x))=\frac{1}{4} \int_{x}^{\infty} d y e^{-(y-x) / 4} y^{n}=4^{n} n ! \sum_{j=0}^{n}(j !)^{-1}(x / 4)^{j}
$$

(i) Obvious.

(ii) This follows by induction on $n_{1}, n_{2}$ when we note that $\lambda_{n}^{\prime}=n \lambda_{n-1}$ and $\lambda_{n_{1}}(0) \lambda_{n_{2}}(0) \leqq \lambda_{n_{1}+n_{2}}(0)$. For then

$$
\left(\lambda_{n_{1}+n_{2}}-\lambda_{n_{1}} \lambda_{n_{2}}\right)^{\prime}=n_{1}\left(\lambda_{n_{1}+n_{2}-1}-\lambda_{n_{1}-1} \lambda_{n_{2}}\right)^{\prime}+n_{2}\left(\lambda_{n_{1}+n_{2}-1}-\lambda_{n_{1}} \lambda_{n_{2}-1}\right)^{\prime} \geqq 0 .
$$

$$
\begin{gathered}
\int_{r}^{\infty} d \zeta e^{-1 / \zeta} \zeta^{-2} E(\zeta)^{-1+\beta} \lambda_{n}(\zeta)=\frac{1}{4} \int_{x}^{\infty} d y_{1} e^{-\beta y_{1}} \int_{y_{1}}^{\infty} d y_{2} e^{-\left(y_{2}-y_{1}\right) / 4} y_{2}^{n} \\
=\frac{e^{-\beta x}}{4} \int_{x}^{\infty} d y_{2} e^{-\left(y_{2}-x\right) / 4} y_{2}^{n}\left[\int_{x}^{y_{2}} d y_{1} e^{-(\beta-1 / 4)\left(y_{1}-x\right)}\right] \\
\leqq\left[\frac{E(r)^{\beta}}{\beta}\right]\left[\frac{\beta}{\beta-1 / 4}\right] \lambda_{n}(r) \leqq 2\left[\frac{E(r)^{\beta}}{\beta}\right] \lambda_{n}(r) \quad \text { if } \beta \geqq 1 / 2 .
\end{gathered}
$$

$$
\begin{aligned}
\int_{0}^{r} d \zeta e^{-1 / \zeta} \zeta^{-2} E(\zeta)^{-1} \lambda_{n}(\zeta) & =\int_{0}^{x} d y \tilde{\lambda}_{n}(y) \\
& =\left.(n+1)^{-1} \tilde{\lambda}_{n+1}\right|_{0} ^{x} \leqq(n+1)^{-1} \tilde{\lambda}_{n+1}(x) .
\end{aligned}
$$

(v) Obvious. QED

Theorem 6. Consider a tree $\tau$ with labelling $\varrho$ contributing to the renormalized effective potential $V_{r}^{\Lambda}(\phi)$. The value $v(r, \phi)=\Sigma_{\mathbf{n}} v_{\mathbf{n}}(r, \phi)$ is bounded term by term, 
uniformly in $\Lambda$ :

$$
\left\|v_{\mathbf{n}}(r)\right\|_{\gamma} \leqq \begin{cases}\lambda^{v} K^{N(\tau)} c(\tau) 2^{\gamma}(\gamma+1)^{-2} \lambda_{\kappa}(r) r^{-\gamma / 2} E(r)^{-\delta_{\mathbf{n}} / 2}, & \text { if } \varrho_{F}=R ; \\ \lambda^{v} K^{N(\tau)} c(\tau) \lambda_{\kappa}(r) r^{\delta_{\mathbf{n}} / 2}, & \text { if } \varrho_{F}=C, \gamma=0 ; \\ 0, & \text { if } \varrho_{F}=C, \gamma>0,\end{cases}
$$

where $\delta_{\mathbf{n}} \leqq-1$ if $\varrho_{F}=R$ and $\delta_{\mathbf{n}} \geqq 0$ if $\varrho_{F}=C$. Here $c(\tau)$ is the combinatoric factor defined by (39) with $\left|\delta_{F}\right|^{-1}$ replaced by $\left[\max \left\{1,-\delta_{F}\right\}\right]^{-1}$. K is a constant, and $\kappa$ is the number of $C^{0}$-forks in $\tau$.

Remarks. (i) It follows that

$$
V_{r}=\lim _{\Lambda \rightarrow \infty} V_{r}^{\Lambda}
$$

exists as a formal power series in the coupling constant $\lambda$.

(ii) Putting $r=0$ and $\gamma=0$ into these bounds, and noting that $\lambda_{\kappa}(0)=4^{\kappa} \kappa$ ! we find

$$
\begin{cases}\left\|v_{\mathbf{n}}(0)\right\|_{0} \leqq \lambda^{v} K^{N(\tau)} c(\tau) 4^{\kappa} \kappa !, & \text { if } \delta_{\mathbf{n}} \leqq 0 \\ \left\|v_{\mathbf{n}}(0)\right\|_{0}=0, & \text { if } \delta_{\mathbf{n}}>0\end{cases}
$$

(iii) The ansatz $2^{\gamma}(\gamma+1)^{-2} r^{-\gamma / 2}$ for the $\gamma$-dependence of (78) is a technical refinement not found in [7] and [4]. The somewhat simpler argument found there leads to bounds which are not uniform as $M \rightarrow 1$, and so fails to extend to the continuous RG approach.

Proof. First, we note the following bounds which hold for all $0 \leqq \zeta<\infty, \beta \geqq 0$ and multiindices $p, q$ with $|p|,|q| \leqq 3$ :

$$
\begin{gathered}
r E(r) \leqq 1, \\
\sum_{p, q} E(\zeta)^{(|p|+|q|) / 2}\left\|\partial^{p} \partial^{q} c(\zeta)\right\|_{\beta} \leqq c_{1} \pi^{2} e^{-1 / \zeta} \zeta^{-2-\beta / 2}, \\
\sum_{p, q} \sup _{y} E(\zeta)^{(|p|+|q|) / 2}\left|\partial^{p} \partial^{q} c(y, z, \zeta)\right| \leqq c_{1} e^{-1 / \zeta}
\end{gathered}
$$

for some constant $c_{1}$.

Suppose $F \in \mathscr{F}_{C^{m}}^{(1)}$ with $m=0$ or 2 . If $v_{\mathbf{n}^{\prime}}=C^{m} v_{\mathbf{n}}$, with $\delta_{\mathbf{n}} \geqq m$, then

$$
\left\|v_{\mathbf{n}^{\prime}}\right\|_{0}=\frac{1}{2}\left\|\int_{0}^{r} C^{m} B_{\mathbf{n}}^{(1)}(\zeta) d \zeta\right\|_{0} \leqq \frac{1}{2} \int_{0}^{r}\left\|B_{\mathbf{n}}^{(1)}(\zeta)\right\|_{\delta_{\mathbf{n}}-m} d \zeta .
$$

From (70) and the formula $\delta_{(\mathbf{n}, p, q)}=\delta_{\mathbf{n}}-|p|-|q|-2$, we calculate

$$
\begin{aligned}
\left\|v_{\mathbf{n}^{\prime}}\right\|_{0} & \leqq \lambda^{v} K^{N-1} d_{1}\left(d_{1}-1\right) c\left(\tau_{1}\right) c_{1} \frac{1}{2} \int_{0}^{r} d \zeta e^{-1 / \zeta} \zeta^{\left(m-\delta_{\mathbf{n}}\right) / 2} \lambda_{\kappa_{1}}(\zeta) E(\zeta)^{\left(2-\delta_{\mathbf{n}}\right) / 2} \\
& \leqq \lambda^{v} K^{N-1} d_{1}\left(d_{1}-1\right) c\left(\tau_{1}\right) c_{1} \frac{1}{2} \int_{0}^{r} d \zeta e^{-1 / \zeta} \zeta^{-2} \zeta^{m / 2} \lambda_{\kappa_{1}}(\zeta) E(\zeta)^{-1}
\end{aligned}
$$

If $m>0$ use of $\zeta^{m / 2} \lambda_{\kappa_{1}}(\zeta) \leqq r^{m / 2} \lambda_{\kappa}(r)$ leads to the desired bound:

$$
\left\|v_{\mathbf{n}^{\prime}}\right\|_{0} \leqq \lambda^{v} c_{1} K^{n-1} c(\tau) \lambda_{\kappa}(r) r^{m / 2}
$$


with $\kappa=\kappa_{1}$. If $m=0$ (73) gives

$$
\left\|v_{\mathbf{n}^{\prime}}\right\|_{0} \leqq \lambda^{v} c_{1} K^{n-1} c(\tau) \lambda_{\kappa}(r)
$$

with $\kappa=\kappa_{1}+1$.

Suppose $F \in \mathscr{F}_{C^{m}}^{(2)}$. Then the identity:

$$
L B^{(2)}\left(v_{1}, v_{2}\right)=L B^{(2)}\left(L v_{1}, L v_{2}\right)
$$

(easy to show) implies that $v(\tau)$ vanishes unless $v\left(\tau_{1}\right)$ and $v\left(\tau_{2}\right)$ are both either trivial $(=V(\phi))$ or $C$-trees. This fact eliminates the $\alpha, \beta$-sum in (69). When we note that $\delta_{\left(\mathbf{n}_{n_{1}}, p\right)}+\delta_{\left(\mathbf{n}_{\pi_{2}}, q\right)}=\delta_{\mathbf{n}}-p-q+2$ and use (82) to do the $p, q$-sum, we find:

$$
\begin{aligned}
\left\|v_{\mathbf{n}^{\prime}}\right\|_{0} \leqq & \frac{1}{2} \int_{0}^{r}\left\|B_{\mathbf{n}}^{(2)}(\zeta)\right\|_{\delta_{\mathbf{n}}-m} d \zeta \leqq \lambda^{v} K^{N-1} d_{1} d_{2} c\left(\tau_{1}\right) c\left(\tau_{2}\right) c_{1} \pi^{2} \sup _{\Pi} \\
& \frac{1}{2} \int_{0}^{r} d \zeta e^{-1 / \zeta} \zeta^{\left(m-4-\delta_{\mathbf{n}}\right) / 2} \lambda_{\kappa_{1}}(\zeta) \lambda_{\kappa_{2}}(\zeta) \zeta^{\left(\delta_{\mathbf{n}}+2\right) / 2}
\end{aligned}
$$

Use of Lemma 5 , and the bound $\zeta^{-1} \leqq \zeta^{-2} E^{-1}$ leads to the required bound

$$
\left\|v_{\mathbf{n}^{\prime}}\right\|_{0} \leqq c_{1} K^{n-1} c(\tau) \lambda_{\kappa}(r) r^{m / 2}
$$

with $\kappa=\kappa_{1}+\kappa_{2}$ if $m>0$ and $\kappa=\kappa_{1}+\kappa_{2}+1$ if $m=0$.

Now suppose $F \in \mathscr{F}_{R}^{(1)}$ and $\delta_{\mathbf{n}} \geqq 0$. From (70) and (83) we find

$$
\begin{aligned}
\left\|v_{\mathbf{n}^{\prime}}\right\|_{\gamma} \leqq & 3 \lambda^{\nu} K^{N-1} d_{1}\left(d_{1}-1\right) c\left(\tau_{1}\right) c_{1} \\
& {\left[\frac{1}{2} \int_{r}^{\infty} d \zeta e^{-1 / \zeta} \frac{2^{\gamma+\delta_{\mathbf{n}}+1}}{\left(\gamma+\delta_{\mathbf{n}}+2\right)^{2}} \zeta^{-\left(\gamma+\delta_{\mathbf{n}}+1\right) / 2} \lambda_{\kappa_{1}}(\zeta) E(\zeta)^{\left(2-\delta_{\mathbf{n}}\right) / 2}\right] . }
\end{aligned}
$$

When we note that $\zeta^{-\left(\delta_{\mathbf{n}}+1\right) / 2} E(\zeta)^{\left(2-\delta_{\mathbf{n}}\right) / 2} \leqq \zeta^{-2} E(\zeta)^{-1 / 2}$ and $\zeta^{-\gamma / 2} \leqq r^{-\gamma / 2}$, we find that the $\zeta$-integral can be done using (74), leading to

$$
\left\|v_{\mathbf{n}^{\prime}}\right\|_{\gamma} \leqq \lambda^{\nu}\left[3 \cdot 2^{3} c_{1}\right] K^{N-1} c(\tau) \frac{2^{\gamma}}{(\gamma+1)^{2}} \lambda_{\kappa}(r) r^{-\gamma / 2} E(r)^{-\delta_{\mathbf{n}^{\prime} / 2}} .
$$

The desired bound is proved easily if $\delta_{\mathbf{n}}<0$.

If $F \in \mathscr{F}_{R}^{(2)}$, then $R=1$ unless both $\tau_{1}$ and $\tau_{2}$ are $C$-trees or are trivial [otherwise, by (88), the counterterm part would vanish]. Thus, in almost all cases no renormalization is needed. If $\delta_{\mathbf{n}}<0$, then $v_{\mathbf{n}^{\prime}} \equiv R v_{\mathbf{n}}=v_{\mathbf{n}}$ and

$$
\begin{aligned}
\left\|v_{\mathbf{n}^{\prime}}\right\|_{\gamma} \leqq & \lambda^{v} K^{N-1} d_{1} d_{2} c\left(\tau_{1}\right) c\left(\tau_{2}\right) c_{1} \pi^{2} \sum_{\beta=0}^{\gamma} \sum_{\alpha=0}^{\gamma-\beta} \frac{2^{\gamma-\beta}}{(\alpha+1)^{2}(\gamma-\beta-\alpha+1)^{2}} \\
& \frac{1}{2}\left[\int_{r}^{\infty} d \zeta e^{-1 / \zeta} \zeta^{-(\beta+4) / 2} \lambda_{\kappa_{1}}(\zeta) \lambda_{\kappa_{2}}(\zeta) \zeta^{-(\gamma-\beta) / 2} E(\zeta)^{-\left(\delta_{\mathbf{n}}+2\right) / 2}\right],
\end{aligned}
$$

where we have used $\delta_{\left(\mathbf{n}_{\pi_{1}}, q\right)}+\delta_{\left(\mathbf{n}_{\pi_{2}}, q\right)}=\delta_{\mathbf{n}}-p-q+2$, and the bound (82) as before. One can show that

$$
\sum_{\beta=0}^{\gamma} \sum_{\alpha=0}^{\gamma-\beta} \frac{2^{\gamma-\beta}}{(\alpha+1)^{2}(\gamma-\beta-\alpha+1)^{2}} \leqq \frac{8 \pi^{2}}{3} \frac{2^{\gamma}}{(\gamma+1)^{2}} .
$$


Putting these results together, we find the desired bound

$$
\left\|v_{\mathbf{n}^{\prime}}\right\|_{\gamma} \leqq \lambda^{\nu}\left[8 \pi^{2} c_{1} / 3\right] K^{N-1} c(\tau) \frac{2^{\gamma}}{(\gamma+1)^{2}} \lambda_{\kappa}(r) r^{-\gamma / 2} E(r)^{-\delta_{\mathbf{n}^{\prime}} / 2} .
$$

Renormalization is needed at $F \in \mathscr{F}_{R}^{(2)}$ only if $\tau_{1}$ and $\tau_{2}$ are $C$-trees. Similarly to the case $F \in \mathscr{F}_{C}^{(2)}$ we have for $\delta_{\mathbf{n}} \geqq 0$

$$
\begin{aligned}
\left\|v_{\mathbf{n}^{\prime}}\right\|_{\gamma} & \leqq \frac{3}{2} \int_{r}^{\infty}\left\|B_{\mathbf{n}}^{(2)}(\zeta)\right\|_{\gamma+\delta_{\mathbf{n}}+1} d \zeta \\
& \leqq \lambda^{\nu} K^{N-1} d_{1} d_{2} c\left(\tau_{1}\right) c\left(\tau_{2}\right) c_{1} \frac{3}{2}\left[\int_{r}^{\infty} d \zeta e^{-1 / \zeta} \zeta^{-(\gamma+1+2) / 2} \lambda_{\kappa_{1}+\kappa_{2}}(\zeta)\right] .
\end{aligned}
$$

Now $\zeta^{-3 / 2} \leqq \zeta^{-2} E^{-1 / 2}$, and so we find

$$
\left\|v_{\mathbf{n}^{\prime}}\right\|_{\gamma} \leqq 3 \lambda^{\nu} K^{N-1} d_{1} d_{2} c\left(\tau_{1}\right) c\left(\tau_{2}\right) c_{1} \zeta^{-\gamma / 2} \lambda_{\kappa}(r) E(r)^{1 / 2}
$$

which is appropriate for $\delta_{\mathbf{n}^{\prime}}=-1$.

This completes the proof provided we take

$$
K=8 \pi^{2} c_{1} / 3 \text {. QED }
$$

Acknowledgements. It is a pleasure to thank Prof. L. Rosen for his helpful comments on this work.

\section{References}

1. Bogoliubov, N.N., Parasiuk, O.S.: Acta Math. 97, 227 (1957)

2. de Calan, C., Rivasseau, V.: Local existence of the Borel transform in Euclidean $\Phi_{4}^{4}$. Commun. Math. Phys. 82, 69-100 (1981)

3. Dyson, F.J.: The radiation theories of Tomonaga, Schwinger, and Feynman. Phys. Rev. 75, 486-502 (1949), and The $S$-matrix in quantum electrodynamics. Phys. Rev. 75, 1736-1755 (1949)

4. Feldman, J.S., Hurd, T.R., Rosen, L., Wright, J.D.: QED: a proof of renormalizability. Lecture Notes in Physics, Vol. 312. Berlin, Heidelberg, New York: Springer 1988

5. Feldman, J., Magnen, J., Rivasseau, V., Sénéor, R.: Bounds on a renormalized Feynman graph, Commun. Math. Phys. 100, 23-55 (1985)

6. Feldman, J., Magnen, J., Rivasseau, V., Sénéor, R.: The massive Gross-Neveu model: a rigorous perturbative construction. Commun. Math. Phys. 103, 67-103 (1986), and Construction and Borel summability of infrared $\phi_{4}^{4}$ by a phase space expansion. Commun. Math. Phys. 109, 437-480 (1987)

7. Gallavotti, G., Nicolò, F.: Renormalization theory in four-dimensional scalar fields. I, II. Commun. Math. Phys. 100, 545-590 (1985), and Commun. Math. Phys. 101, 247-282 (1986)

8. Gawedzki, K., Kupiainen, A.: Massless lattice $\phi_{4}^{4}$ theory: rigorous control of a renormalizable asymptotically free model. Commun. Math. Phys. 99, 197-252 (1985), and Gross-Neveu model through convergent perturbation expansions. Commun. Math. Phys. 102, 1-30 (1985)

9. Hepp, K.: Proof of the Bogoliubov-Parasiuk theorem on renormalization. Commun. Math. Phys. 2, 301-326 (1966)

10. Polchinski, J.: Renormalization and effective lagrangians. Nucl. Phys. B231, 269-295 (1984)

11. Weinberg, S. : High energy behavior in quantum field theory. Phys. Rev. 118, 838-849 (1960) 
12. Wilson, K.G.: Renormalization group and strong interactions. Phys. Rev. D3, 1818-1846 (1971), and Renormalization of a scalar field theory in strong coupling. Phys. Rev. D6, 419-426 (1972)

13. Wilson, K.G., Kogut, J.: The renormalization group and the $\varepsilon$-expansion. Phys. Rep. 12, 75-200 (1974)

14. Zimmermann, W.: Convergence of Bogoliubov's method of renormalization in momentum space. Commun. Math. Phys. 15, 208-234 (1969)

Communicated by K. Gawedzki

Received January 16, 1989 